\title{
PERCEPCIÓN DE LA VIOLENCIA DURANTE LA PRÁCTICA DE ACTIVIDADES FÍSICAS EN ESCOLARES
}

PERCEIVED VIOLENT CLIMATE DURING PHYSICAL ACTIVITY IN SCHOOL-AGED YOUTH

\author{
Oswaldo Ceballos-Gurrola ${ }^{1}$ \\ Rosa Elena Medina-Rodríguez ${ }^{2}$ \\ Jeanette Magnolia López-Walle ${ }^{3}$ \\ José Leandro Tristán-Rodríguez ${ }^{4}$
}

\section{Resumen}

El propósito del presente trabajo es brindar información hacia los docentes, escolares, padres de familia y público en general sobre la práctica físico-deportiva desarrollada en la escuela y su incidencia en la violencia de género y contra grupos vulnerables. Se puede señalar que la violencia hacia la mujer y los grupos vulnerables es un problema social que está presente en la vida diaria de las personas, aun en el ámbito escolar entre los niños y las niñas, los que tienen obesidad, capacidades especiales o falta de habilidad para las tareas habituales.

Se describen los resultados a través de un diagnóstico de la práctica físico-deportiva y los problemas antes mencionados, se presentan conclusiones y recomendaciones para dar solución a los problemas detectados, como identificar las causas de la violencia y darles un tiempo a los involucrados para que reflexionen y lleguen a un acuerdo para evitar este tipo de conductas, ofrecer entrenamiento en resolución de conflictos, educar en valores democráticos, de tolerancia y de respeto intercultural.

Palabras claves: violencia, mujeres, grupos vulnerables, actividades físico-deportivas, escolares

\section{Abstract}

The purpose of this paper is to provide teachers, students, parents, and communities with information on sports and physical activities carried out in school environments, and their impact on gender violence and vulnerable groups. Violence against women and vulnerable groups is a well-known social issue that is spread in people's day-by-day life. This situation can even be found in schools, especially against children suffering from obesity, needing special care, or being less skilled in common school tasks.

After executing a diagnosis on sports and physical skills and on the aforementioned variables, recommendations are provided in order to detect the causes of violence; for example, make persons involved in violent acts consider their consequences; find measures for avoiding this type of behaviours; provide training on conflict solving methods; and educate in democratic values, tolerance and intercultural respect.

Keywords: violence, women, vulnerable groups, sports, physical and sport activities, school-aged youth

Fecha de recepción: 15 de julio de 2014

Fecha de aprobación: 12 de noviembre de 2014

1 Doctor en Ciencias de la Actividad Física y del Deporte, por la Universidad de Zaragoza, España. Máster en Ciencias del Ejercicio, con especialidad en Educación Física y Deporte en la Infancia y la Adolescencia, por la Facultad de Organización Deportiva de la Universidad Autónoma de Nuevo León. Licenciado en Organización Deportiva de la Facultad de Organización Deportiva de la Universidad Autónoma de Nuevo León. Director de la Facultad de Organización Deportiva, Universidad Autónoma de Nuevo León. Correo electrónico: oscegu@hotmail.com

2 Doctora en Ciencias Económicas y Empresariales en la Universidad de Zaragoza, España. Máster en Marketing y Gestión Comercial del Departamento de Economía y Dirección de Empresas y el Club de Marketing Dirigentes de Aragón, Zaragoza, España. Licenciada en Contador Público y Auditor del Centro de Estudios Universitarios, Monterrey, Nuevo León, México (UANL). Vicepresidente de la Asociación Latinoamericana de Gerencia Deportiva, Algede. Responsable del Área de Gestión Deportiva en el Posgrado de FoD, Universidad Autónoma de Nuevo León. Correo electrónico: rosa.medinard@uanl.edu.mx

3 Posdoctora en Psicología del Deporte. Doctora en Actividad Física y Salud de la Universidad de Granada, España. Maestría en Ciencias Aplicadas al Deporte en la Universidad del Valle de México. Licenciada en Psicología por la Universidad Nacional Autónoma de México. Profesora investigadora exclusiva de tiempo completo titular "A", subdirectora de Posgrado, Facultad de Organización Deportiva, Universidad Autónoma de Nuevo León. Correo electrónico: jeanette.lopezwl@uanl.edu.mx

4 Doctor en Actividad Física y Calidad de Vida por la Universidad de Murcia, España. Maestría en Ciencias del Ejercicio con Especialidad en Alto Rendimiento (UANL). Licenciado de la Facultad de Organización Deportiva (UANL). Profesor investigador de tiempo completo titular "A" Facultad de Organización Deportiva, Universidad Autónoma de Nuevo León (México). 
Para citar este artículo:

Ceballos-Gurrola, O., Medina-Rodríguez, R.E., López-Walle,

J.M., Tristán-Rodríguez, J.L. (2014). Percepción de la violencia durante la práctica de actividades físicas en escolares. Revista Lúdica Pedagógica, (20), 87-92.

\section{INTRODUCCIÓN}

La violencia hacia la mujer y los grupos vulnerables es un problema social que está presente en la vida diaria de las personas, aun en el ámbito escolar entre los niños y las niñas, los que presentan obesidad, discapacidad o falta de habilidad para las actividades físico-deportivas.

La Secretaría de Educación y la Universidad Autónoma de Nuevo León, por intermedio del Centro de Investigación para la Comunicación, preocupados por los diferentes problemas sociales, proponen el Programa de Capacitación al Magisterio para Prevenir la Violencia hacia las Mujeres. Como parte de este programa se diseñó una línea de acción hacia la "práctica físico-deportiva desarrollada en la escuela: violencia de género y grupos vulnerables" como una estrategia importante de diagnóstico, capacitación y propuesta de soluciones con este enfoque.

La violencia contra la mujer es definida por la Organización de las Naciones Unidas (ONU, 2014) como:

... todo acto de violencia basado en la pertenencia al sexo femenino que tenga o pueda tener como resultado un daño o sufrimiento físico, sexual o sicológico para la mujer, así como las amenazas de tales actos, la coacción o la privación arbitraria de la libertad, tanto si se producen en la vida pública como en la vida privada.

La violencia de género durante la práctica físico-deportiva se ve reflejada en diferentes momentos, en los cuales los hombres la utilizan con mayor frecuencia e intensidad que las mujeres (Rutter, Giller y Hagel, 1998), diferencia que se manifiesta desde la infancia en cualquier contexto, incluido el escolar (Hernando, 2007; Olafsen y Viemero, 2000), y que, como sucede en otros contextos y edades, cabe relacionar con los estereotipos masculinos tradicionales, en los que se asocia el valor del hombre con el dominio, con el control absoluto y con la violencia (Cowie, 2000; Díaz-Aguado y Martínez-Arias, 2001). Los análisis realizados sobre la frecuencia con la que se sufren en la escuela situaciones de victimización en función del género encuentran resultados menos consistentes (Olafsen y Viemero, 2000; Smith y Sharp, 1994).
Para explicar la relación entre agresión y género, conviene tener en cuenta que la principal condición de riesgo de violencia no es el sexo como condición biológica, sino la identificación con el dominio de los demás, asociada al estereotipo masculino tradicional. Esta identificación incrementa el riesgo de convertirse en agresor de los compañeros tanto en los hombres, entre los que suele ser más frecuente, como en las mujeres (Young y Sweeting, 2004).

Por otra parte, en diversas investigaciones también se pone de manifiesto la necesidad de considerar diferencias cualitativas, puesto que, por ejemplo, la utilización de la violencia por las mujeres suele ser mucho más indirecta (Österman, Björkqvist y Lagerspezt, 1998) y está influida por presiones situacionales con mayor intensidad (Salmivalli, Lappalainen y Lagerspetz,1998).

En un estudio Díaz-Aguado, Martínez-Arias y Martín-Seoane (2004) encuentran, así mismo, que los hombres manifiestan en casi todos los indicadores evaluados un riesgo superior de violencia y de intolerancia que las mujeres. La ausencia de diferencias de género en el ocio puede ser interpretada como un indicador de la fuerte presión grupal que suele existir en dicho contexto, que podría obstaculizar los mecanismos de inhibición de la violencia en las adolescentes, lo que refleja la necesidad de orientar la superación del sexismo de forma que favorezca una identificación con la totalidad de los valores tradicionalmente asociados a lo femenino (la empatía, la ternura) y a lo masculino (la fuerza y el poder), sin que nadie tenga que identificarse con los problemas a los que dichos estereotipos conducen (como la violencia).

Entre las características más frecuentes observadas en los alumnos que acosan a sus compañeros, destacan las siguientes (Pellegrini, Bartini y Brooks, 1999): una situación social negativa, aunque cuentan con algunos amigos que les siguen en su conducta violenta; una acentuada tendencia a abusar de su fuerza (suelen ser físicamente más fornidos que los demás); son impulsivos, con escasas habilidades sociales, con baja tolerancia a la frustración, y con dificultad para cumplir normas; unas relaciones negativas con relación a los adultos y un bajo rendimiento, problemas que se incrementan con la edad y de los hombres hacia las mujeres; no son muy autocríticos, por lo que cabe considerar el hecho observado en varias investigaciones al intentar evaluar la autoestima de los agresores y encontrarla media o incluso alta.

El propósito del presente artículo es brindar información hacia el magisterio, escolares, padres de familia 
y público en general sobre la práctica físico-deportiva desarrollada en la escuela y su incidencia en la violencia de género y grupos vulnerables.

\section{MÉTODO}

\section{Participantes}

Se realizó un estudio no probabilístico y de muestreo por cuotas para escolares de primaria y secundaria de escuelas públicas de las zonas rural y urbana del estado de Nuevo León, a los que se les aplicó una encuesta enfocada al análisis temático y de forma descriptiva y analítica.

Los datos demográficos de los escolares que se incluyeron en el estudio muestran una participación de 703 con un error de $\pm 4 \%$, de los cuales 339 son hombres y 364 mujeres, con un promedio de edad de 11.47 años; de estos, 404 pertenecen a la zona urbana y 299 a la rural.

\section{Instrumento}

Para determinar la violencia hacia las mujeres y los grupos vulnerables durante la práctica de actividades físico-deportivas se diseñó una encuesta específica para este estudio, la cual fue validada por expertos y consta de 30 reactivos relacionados con el tema de estudio. La instrucción general dice "Marca con una X en el recuadro, según la frecuencia con que se han presentado algunos de los siguientes acontecimientos durante la clase de Educación Física, recreo, hora libre, entrada y salida de clases"; se ofrecen cuatro opciones de respuesta para cada reactivo: Siempre, Casi siempre, Algunas veces y Nunca.

Las encuestas las aplicaron personas capacitadas para ello, siempre con el consentimiento de las autoridades educativas y los padres de familia. Se tomaron grupos de diez estudiantes y en un salón de clase se impartieron las instrucciones básicas y respuestas concretas a las posibles dudas para no influir en las mismas.

Una vez recogidos, los datos fueron analizados mediante el paquete informático SPSS v 16 , se obtuvieron estadísticas descriptivas mediante frecuencias y porcentajes, así como la comparación de los valores según género en las áreas rural y urbana mediante tablas de contingencia.

\section{Resultados}

En general, los hombres presentan conductas de violencia verbal hacia las mujeres y los compañeros del mismo sexo (Tabla 1). Es una costumbre tanto en el área urbana como en la rural el utilizar palabras que ofenden a sus compañeros (87\%), al igual que actitudes burlarse de sus compañeros cuando ganan un juego (61\%).

Tanto en el área urbana como en la rural, y en esta última con más frecuencia entre los hombres $(\mathrm{P}=.016)$, es una actitud habitual que los escolares agredan a un compañero al estar practicando actividades físico-deportivas (Tabla 2). Así mismo, los hombres son los que preferentemente realizan juegos que son muy bruscos

Tabla 1. Frecuencias y porcentajes de escolares que utilizan palabras que ofenden a sus compañeros.

\begin{tabular}{|c|c|c|c|c|c|}
\hline \multirow{2}{*}{\multicolumn{2}{|c|}{ Utilizan palabras que ofenden }} & \multicolumn{2}{|c|}{ Área urbana } & \multicolumn{2}{|c|}{ Área rural } \\
\hline & & Hombres & Mujeres & Hombres & Mujeres \\
\hline \multirow{2}{*}{ No contesta } & $\mathbf{N}$ & 1 & 1 & 0 & 0 \\
\hline & $\%$ & $.5 \%$ & $.5 \%$ & 0 & 0 \\
\hline \multirow{2}{*}{ Siempre } & $\mathbf{N}$ & 7 & 3 & 11 & 1 \\
\hline & $\%$ & $3.6 \%$ & $1.4 \%$ & $7.5 \%$ & $.7 \%$ \\
\hline \multirow{2}{*}{ Con frecuencia } & $\mathbf{N}$ & 13 & 8 & 5 & 5 \\
\hline & $\%$ & $6.7 \%$ & $3.8 \%$ & $3.4 \%$ & $3.3 \%$ \\
\hline \multirow{2}{*}{ Algunas veces } & $\mathbf{N}$ & 93 & 74 & 79 & 66 \\
\hline & $\%$ & $47.9 \%$ & $35.2 \%$ & $54.1 \%$ & $43.1 \%$ \\
\hline \multirow{2}{*}{ Nunca } & $\mathbf{N}$ & 80 & 124 & 51 & 81 \\
\hline & $\%$ & $41.2 \%$ & $59.0 \%$ & $34.9 \%$ & $52.9 \%$ \\
\hline \multicolumn{2}{|c|}{ P valor } & \multicolumn{2}{|c|}{.008} & \multicolumn{2}{|c|}{.001} \\
\hline
\end{tabular}


Tabla 2. Frecuencias y porcentajes de escolares que han agredido a un compañero al estar practicando actividades físico-deportivas

\begin{tabular}{|c|c|c|c|c|c|}
\hline \multirow{2}{*}{\multicolumn{2}{|c|}{ Han castigado a un compañero }} & \multicolumn{2}{|c|}{ Área urbana } & \multicolumn{2}{|c|}{ Área rural } \\
\hline & & Hombres & Mujeres & Hombres & Mujeres \\
\hline \multirow{2}{*}{ No contesta } & $\mathbf{N}$ & 1 & 4 & 2 & 0 \\
\hline & $\%$ & $.5 \%$ & $1.9 \%$ & $1.4 \%$ & $.0 \%$ \\
\hline \multirow{2}{*}{ Siempre } & $\mathbf{N}$ & 8 & 5 & 10 & 6 \\
\hline & $\%$ & $4.1 \%$ & $2.4 \%$ & $6.8 \%$ & $3.9 \%$ \\
\hline \multirow{2}{*}{ Con frecuencia } & $\mathbf{N}$ & 15 & 6 & 13 & 4 \\
\hline & $\%$ & $7.7 \%$ & $2.9 \%$ & $8.9 \%$ & $2.6 \%$ \\
\hline \multirow{2}{*}{ Algunas veces } & $\mathbf{N}$ & 86 & 88 & 65 & 62 \\
\hline & $\%$ & $44.3 \%$ & $41.9 \%$ & $44.5 \%$ & $40.5 \%$ \\
\hline \multirow{2}{*}{ Nunca } & $\mathbf{N}$ & 84 & 107 & 56 & 81 \\
\hline & $\%$ & $43.3 \%$ & $51.0 \%$ & $38.4 \%$ & $52.9 \%$ \\
\hline \multicolumn{2}{|c|}{$P$ valor } & \multicolumn{2}{|c|}{.074} & \multicolumn{2}{|c|}{.016} \\
\hline
\end{tabular}

y que lastiman a sus compañeros (46\%), ven programas violentos y disfrutan repetirlos con sus colegas (68\%).

La violencia emocional está presente durante la práctica de actividades físico-deportivas de los escolares. Un ejemplo de ello es que los hombres y las mujeres coinciden en que cuando pierden un juego o partido, les da coraje y agreden física o verbalmente a sus compañeros (27 \%).
En otras ocasiones, los hombres son los que ridiculizan habitualmente a sus compañeros diciéndoles que actúan como niñas (34\%), se burlan de los compañeros que son diferentes por su aspecto (especialmente los hombres del área urbana, 30\%) o por su forma de hablar (20\%). Como respuesta a estas y otras actitudes negativas, no se les da permiso de jugar durante el recreo ( $41 \%$ en hombres y $28 \%$ en mujeres).

Tabla 3. Frecuencias y porcentajes de escolares a quienes no les gusta juntar a las niñas o a otros niños

\begin{tabular}{|c|c|c|c|c|c|}
\hline \multirow{2}{*}{\multicolumn{2}{|c|}{ No les gusta juntar a las niñas }} & \multicolumn{2}{|c|}{ Área urbana } & \multicolumn{2}{|c|}{ Área rural } \\
\hline & & Hombres & Mujeres & Hombres & Mujeres \\
\hline \multirow{2}{*}{ No contesta } & $\mathbf{N}$ & 1 & 3 & 1 & 4 \\
\hline & $\%$ & $.5 \%$ & $1.4 \%$ & $.7 \%$ & $2.6 \%$ \\
\hline \multirow{2}{*}{ Siempre } & $\mathbf{N}$ & 30 & 33 & 22 & 26 \\
\hline & $\%$ & $15.5 \%$ & $15.7 \%$ & $15.1 \%$ & $17.0 \%$ \\
\hline \multirow{2}{*}{ Con frecuencia } & $\mathbf{N}$ & 13 & 11 & 10 & 2 \\
\hline & $\%$ & $6.7 \%$ & $5.2 \%$ & $6.8 \%$ & $1.3 \%$ \\
\hline \multirow{2}{*}{ Algunas veces } & $\mathbf{N}$ & 63 & 39 & 46 & 38 \\
\hline & $\%$ & $32.5 \%$ & $18.6 \%$ & $31.5 \%$ & $24.8 \%$ \\
\hline \multirow{2}{*}{ Nunca } & $\mathbf{N}$ & 87 & 124 & 67 & 83 \\
\hline & $\%$ & $44.8 \%$ & $59.0 \%$ & $45.9 \%$ & $54.2 \%$ \\
\hline \multicolumn{2}{|c|}{$P$ valor } & \multicolumn{2}{|c|}{.012} & \multicolumn{2}{|c|}{.044} \\
\hline
\end{tabular}


Es más común observar la violencia de género de los hombres hacia las mujeres en actitudes como que no les guste juntar a las niñas en juegos o actividades deportivas (55\%) (Tabla 3), así como no juntar a otros niños porque no son buenos para jugar (48\%).

Los escolares opinan que el espacio y el material que se les asigna para jugar tanto a los niños como a las niñas son equitativos $(\mathrm{P}>0.05)$. Es más evidente que tanto a las niñas (34\%) como a los niños (45\%) del área rural no les gusta jugar con niños que tienen alguna discapacidad o impedimento físico, son obesos (59.5\% y $57.5 \%$ respectivamente), son morenos (38\% y $41 \%$ respectivamente), y además suelen molestar a alumnos de grados inferiores ( $85 \%$ y $68 \%$ respectivamente).

\section{Discusión}

Los datos muestran que existen diferentes tipos de violencia durante la práctica de actividades físico-deportivas en el entorno escolar, siendo más representativas las verbales, físicas y de discriminación. Existe otro estudio que muestra resultados similares en centros escolares de educación secundaria de España (Álvarez-García et ál., 2007), siendo más frecuente la violencia verbal entre los mismos compañeros que la dirigida al profesorado, con acciones como poner motes (apodos), insultos, hablar mal; la violencia física se presenta con menor frecuencia.

Entre los principales antecedentes familiares, se destacan: la ausencia de una relación afectiva, cálida y segura por parte de los padres, sobre todo de la madre, que manifiesta actitudes negativas o escasa disponibilidad para atender al niño; y fuertes dificultades para enseñar a respetar límites, combinando la permisividad ante conductas antisociales con el frecuente empleo de métodos autoritarios y coercitivos, utilizando en muchos casos el castigo corporal.

La violencia observada en el entorno escolar, en especial hacia las mujeres y los grupos vulnerables ponen de manifiesto la importancia que tiene erradicar situaciones de exclusión desde las primeras etapas educativas, y favorecer la identificación de los adolescentes con los valores de respeto mutuo, de empatía y de no violencia para prevenir el acoso entre escolares (Alberdi y Rojas, 2005).

Cuando la valoración de los programas de prevención la realizan los equipos directivos de los centros, destacan por su eficacia, en primer lugar, los que buscan cambiar la escuela para adaptarla mejor a las necesidades de los alumnos; en segundo lugar, los que promueven cambios y habilidades en el profesorado para prevenir la violencia; $y$, en tercer lugar, los que simplemente pretenden modificar la conducta individual de los alumnos violentos (Heerboth, 2000).

En tal sentido cabe destacar, por ejemplo, la eficacia comprobada en escuelas de primaria de los programas de construcción de la paz aplicados de forma generalizada y continuada, en los que, junto al entrenamiento en resolución de conflictos, se educa en valores democráticos, de tolerancia y de respeto intercultural (Aber y Jones, 2003).

Para lograr prevenir la violencia en el entorno escolar y con especial atención hacia las mujeres, es necesario establecer protocolos de intervención que ayuden a conseguirlo, simplificando sus componentes básicos.

\section{CONCLUSIONES}

Aunque no se puede generalizar, existe un porcentaje elevado de escolares hombres que presentan algún tipo de violencia y como consecuencia reciben más agresiones de sus padres, docentes y compañeros. Por otro lado, suelen agredir a sus compañeras, realizan juegos que son muy bruscos y que lastiman a los demás, ven programas violentos y les gusta repetirlos con sus colegas, cuando pierden un juego o partido les da coraje y agreden física o verbalmente, se burlan de los compañeros que son diferentes por su aspecto o porque son diferentes por su forma de hablar. Lo anterior pone de manifiesto la importancia que tiene erradicar situaciones de exclusión desde las primeras etapas educativas, y favorecer la identificación de los adolescentes con los valores de respeto mutuo, de empatía y de no violencia para prevenir el acoso entre escolares.

Durante la práctica de actividades físico-deportivas de los escolares se presentan diferentes tipos de agresión: verbal, física, emocional y de género; resulta importante identificar las causas dándoles un tiempo a los involucrados para que reflexionen y lleguen a un acuerdo para evitar este tipo de conductas, ofrecer entrenamiento en resolución de conflictos, así como educar en valores democráticos, de tolerancia y de respeto intercultural.

También es importante que los profesores eviten mostrar actitudes estereotipadas y proporcionen ambientes de igualdad de género siempre que sea posible, eviten el enfrentamiento entre hombres y mujeres en clases mixtas, utilicen juegos cooperativos, y garantizar el tiempo y la dedicación necesarios a cada actividad. 


\section{REFERENCIAS BIBLIOGRÁFICAS}

Aber, L. y Jones, S. (2003). Developmental trajectories toward violence in middle childhood: Course, demographic differences, and response to school-based intervention. Developmental Psychology, 39 (2), 324-348.

Alberdi, I. y Rojas, L. (2005). Violencia tolerancia cero. Barcelona: Fundación La Caixa.

Álvarez, D., Álvarez, L., Núñez, J. C., González, J.A., González, P. y Rodríguez, C. (2007). Estudio del nivel de violencia escolar en siete centros asturianos de educación secundaria. Aula Abierta, 36 (1-2), 89-96.

Cowie, H. (2000). Bystanding or standing by: Gender issues in coping with bullying in schools. Aggressive Behavior, 26, 85-97.

Díaz-Aguado, M. J. y Martínez-Arias, R. (2001). La construcción de la igualdad y la prevención de la violencia contra la mujer desde la educación secundaria. Madrid: Instituto de la Mujer, Ministerio de Trabajo y Asuntos Sociales.

Díaz-Aguado, M. J., Martínez-Arias, R. y Martín-Seoane, G. (2004). Prevención de la violencia y lucha contra la exclusión desde la adolescencia, volumen uno: La violencia entre iguales en la escuela y en el ocio. Estudios comparativos e instrumentos de evaluación. Madrid: Instituto de la Juventud.

Heerboth, J. (2000). School violence prevention programs in Southern Illinois high Schools: Factors related to principal's and counselor's perceptions of success, dissertation abstracts international section A: Humanities and Social Sciences, 60 (8-A), 2752.
Hernando, A. (2007). La prevención de la violencia de género en adolescentes. Una experiencia en el ámbito educativo. Apuntes de Psicología, 25 (3), 325-240.

Olafsen R. y Viemero V. (2000). Bully/victim problems in coping with stress. En school among 10-to12 years old pupils in Åland, Finland. Aggressive Behavior, 26, 57-65.

ONU (2014). Organización de las Naciones Unidas. Temas mundiales. Eliminación de la violencia. Recuperado de http://www.un.org/es/globalissues/ women/violencia.shtml

Österman, D., Björkqvist, D. y Lagerspezt, K. (1998). Cross-cultural evidence of female indirect aggression. Aggressive Behavior, 24, 1-8.

Pellegrini, A., Bartini, M. y Brooks, F. (1999). School bullyes, Victims and aggressive victims. Journal of Educational Psychology, 91, 216-224.

Rutter, M., Giller, H. y Hagel, A. (1998). Antisocial behavior in young people, Cambridge: Cambridge University Press.

Salmivalli, C., Lappalainen, M. y Lagerspetz, M. (1998). Stability and change of behavior in connection with bullying in schools: A two-year follow-up. Aggressive Behavior, 24, 205-218.

Smith, P. y Sharp, S. (1994). School bullying. Insights and perspectives, Nueva York: Routledge.

Young, R. y Sweeting, H. (2004). Adolescent bullying, relationships, psychological well-being, and gender-atypical behavior: A gender diagnosticity approach. Sex Roles, 50 (7-8), 525-555. 